\title{
Protostellar collapse models of prolate molecular cloud cores
}

\author{
L. Di G. Sigalotti ${ }^{1}$ and J. Klapp ${ }^{2}$ \\ ${ }^{1}$ Centro de Física, Instituto Venezolano de Investigaciones Científicas, IVIC, Apartado 21827, Caracas 1020A, \\ Venezuela \\ 2 Instituto Nacional de Investigaciones Nucleares, ININ Km. 36.5, Carretera México-Toluca, Ocoyoacac 52045, \\ Estado de México, México \\ e-mail: klapp@nuclear.inin.mx
}

Received 22 June 2001 / Accepted 22 August 2001

\begin{abstract}
The continued detection of binary systems among pre-main-sequence stars suggests that fragmentation is a very frequent process during the early stages of star formation. However, the fragmentation hypothesis rests only upon the results of three-dimensional hydrodynamics code calculations. The validity of isothermal fragmentation calculations was questioned by the results of Truelove et al. (1997), and more recently, of Boss et al. (2000), who found, working at very high spatial resolution, that a particular Gaussian cloud model collapsed isothermally to form a singular filament rather than a binary or quadruple protostellar system as predicted by previous calculations. Sufficiently high spatial resolution is necessary to resolve the Jeans length and hence avoid artificial fragmentation in isothermal collapse calculations. Here we use an adaptive, spherical-coordinate hydrodynamics code based on the "zooming" coordinates to investigate the isothermal collapse of centrally condensed (Gaussian), prolate (2:1 axial ratio) cloud core models, with thermal energy $\alpha \approx 0.22$ and varied rotational energy $(0.246 \leq \beta \leq 0.00025)$, to discern whether they will still undergo fragmentation into a protostellar binary system, as found in most previous prolate cloud collapse calculations, or condense all the way into a thin filament, as suggested by the linear analysis of Inutsuka \& Miyama (1992) and the findings of Truelove et al. and Boss et al. for the spherical, Gaussian cloud model. The prolate clouds all collapsed self-similarly to produce an intermediate barlike core, which then shrank indefinetely into a singular filament without fragmenting. Collapse of the bar into a thin filament also occurred self-similarly, with the forming filaments being much longer than the Jeans length. Since the filaments form at maximum densities that are typical of the transition from the isothermal to the nonisothermal phase, gradual heating may retard the collapse and allow fragmentation of the filament into a binary or multiple protostellar core, as required to explain the high frequency of binary stars.
\end{abstract}

Key words. hydrodynamics - methods: numerical - stars: formation - binaries: general

\section{Introduction}

Observations of star-forming regions show that many dark clouds are filamentary in appearance (Schneider \& Elmegreen 1979; Heyer et al. 1987; Tatematsu et al. 1993; Onishi et al. 1996). Filamentary molecular clouds typically consist of discrete clumps, or cloud cores, many of which are known to contain embedded infrared sources (Dutrey et al. 1991). Considering that self-gravitating filaments are unstable toward fragmentation, several authors have advanced the hypothesis that star-forming cores form through a hierarchical fragmentation process, in which filaments form out of larger structures, and then clumps out of the filaments (Hanawa et al. 1994; Matsumoto et al. 1997; Fiege \& Pudritz 2000; Curry 2000). In this

Send offprint requests to: L. Di G. Sigalotti, e-mail: lsigalot@einstein.ivic.ve scenario, star formation ultimately involves the collapse of cloud cores from an initial density $<10^{-19} \mathrm{~g} \mathrm{~cm}^{-3}$ and size $\approx 10^{17} \mathrm{~cm}$ to a final young stellar object of density $>10^{-1} \mathrm{~g} \mathrm{~cm}^{-3}$ and size $\approx 10^{11} \mathrm{~cm}$. The collapse of a portion of the core may lead to fragmentation, which appears to be a necessary mechanism to establish the observed distributions of mass ratios, periods, and orbital eccentricities of binary stars.

The properties of precollapse (starless) cores are of great importance because they provide true initial conditions for the protostellar collapse phase. Highresolution telescopic observations of starless cores by Ward-Thompson et al. (1994), André et al. (1996), and André et al. (1998) reveal that the radial density profile of the core region is relatively steep $\left(\rho \propto r^{-2}\right)$ toward its outer edge but flattens near its center, similar to the density profiles predicted by calculations of magnetized cores 
in the ambipolar diffusion stage (Fiedler \& Mouschovias 1993; Basu \& Mouschovias 1994). Flat inner density profiles are also characteristic of precollapse cores in clusterforming regions (Motte et al. 1998; Motte \& André 1998). Another important property of precollapse dense cores is their three-dimensional shape. In particular, Myers et al. (1991) mapped 16 dense cores, finding them to be elongated similarly in three different molecular lines. They deduced by statistical arguments that these apparent elongations would indicate prolate, rather than oblate, shapes. If most of these cores are prolate spheroids, their relative elongations would correspond to axial ratios of $\sim 2: 1$, but if some of them are oblate, then their relative flattening would be indicative of axial ratios of $\sim 3: 1$ to $10: 1$, or even larger. Ryden (1996) analyzed the distribution of axial ratios for a large sample of Bok globules and cloud cores taken from several independent data sets. She found that prolate shapes are almost always consistent with the observed axial ratios, and inferred a mean axial ratio for cores of $\sim 2: 1$, in agreement with Myers el al. (1991). Similar degrees of elongation were recently derived by analyzing a sample of dense cores in the line intensity maps of $\mathrm{NH}_{3}$ and CS (Jijina et al. 1999). A brief review of the properties of star-forming cloud cores is given by Myers (1998). Evidence for rotation in molecular cloud cores has been reported by Goodman et al. (1993). They detected rotational motion in 29 cores out of 43 cases studied within the size range $0.06-0.6 \mathrm{pc}$. These detections were recently updated by Barranco \& Goodman (1998), who measured angular velocities in the range $10^{-14}-10^{-13} \mathrm{~s}^{-1}$ and ratios of the rotational kinetic energy to the absolute value of the gravitational energy $\beta$ ranging from 0.001 to 0.03 . Although rotation is dynamically unimportant in molecular cloud cores, it still plays a remarkable role once a core collapses to form a single star or binary system with associated disks (Bodenheimer 1995). The parameters of the newly formed binaries will all depend on the range of angular momenta among the different cores as well as on the angular momentum distributions within individual cores.

In the last decade, fragmentation during the dynamic collapse of cloud cores has emerged as the likely mechanism for explaining the detected binary frequency among pre-main-sequence (PMS) and main-sequence (MS) stars; see recent reviews by Bodenheimer et al. (2000) and Sigalotti \& Klapp (2001). However, studying the fragmentation process requires the use of three-dimensional hydrodynamical collapse calculations, which must rely upon convergence testing and intercode comparisons to validate their results. In particular, the reliability of isothermal collapse calculations has recently been questioned by the results of Truelove et al. (1997), who found employing an adaptive mesh refinement (AMR) code, written in Cartesian coordinates, that a Gaussian cloud model did not fragment into a binary or quadruple system as in previous calculations (Boss 1991, 1993; Klapp et al. 1993; Burkert \& Bodenheimer 1996) but, rather, collapsed into a singular filament consistent with the self-similar solution derived by Inutsuka \& Miyama (1992) for the col- lapse of isothermal cylinders. In short, Truelove at al. (1997) discovered that finite-difference errors may induce "artificial" fragmentation in isothermal collapse calculations for which the Cartesian cell size $\Delta x$ exceeds onefourth of the local Jeans length $\lambda_{\mathrm{J}}$. While this result applies to locally uniform Cartesian grids, Boss (1998) found that for non-uniform spherical coordinate grids the Jeans mass constraint translates into requiring that any of the four lengths $\left[\Delta r, r \Delta \theta, r \sin \theta \Delta \phi,\left(r^{2} \sin \theta \Delta r \Delta \theta \Delta \phi\right)^{1 / 3}\right]$ is less than $\lambda_{\mathrm{J}} / 4$. Recent intercomparison calculations by Boss et al. (2000) for the same Gaussian cloud model of Truelove et al. (1997), have shown that satisfying the Jeans condition at the location of the maximum density, combined with sufficiently high spatial resolution, is necessary to change the nature of the solution from a binary to a singular filament in spherical-grid based calculations. High resolution is therefore crucial to produce reliable results of the collapse and fragmentation of molecular cloud cores.

Previous calculations of the isothermal collapse of prolate clouds not adhering to the Jeans condition, starting with either uniform-density profiles (Nelson \& Papaloizou 1993) or Gaussian condensations (Boss 1993; Boss \& Myhill 1995; Bonnell et al. 1996; Sigalotti \& Klapp 1997; Sigalotti 1998a), have predicted fragmentation into binary systems for a wide range of the initial conditions. In this paper we recalculate the isothermal collapse of prolate core models using high spatial resolution to discern whether they will still undergo fragmentation into a protostellar binary system or condense into a singularity without fragmenting. All models considered were previously known to produce binary systems at low spatial resolution. The calculations were made using a sphericalcoordinate based code as described in Sigalotti (1998b) by adopting "zooming" coordinates to achieve the required high spatial resolution. These coordinates were previously used by Matsumoto \& Hanawa (1999) to study disk and bar formation during the isothermal collapse phase of a molecular cloud core up to extremely high density contrasts. With the zooming coordinates, the computational cells in the ordinary space shrink adaptively in accordance with the cloud collapse. In this way, the four Jeans conditions introduced by Boss (1998) are automatically satisfied because the spatial resolution increases in proportion to the Jeans length as the density enhances. With respect to the AMR framework and other redefinition techniques, the zooming coordinates provide an effective refinement method, which works at a much lower computational cost and without any detailed control. In Sect. 2 we present the transformed hydrodynamic equations to be solved and describe briefly the numerical methods. In Sect. 3 we test the new methods and present a further intercode comparison for the same Gaussian cloud model of Truelove et al. (1997) and Boss et al. (2000). The initial conditions and results for the prolate collapse models are given in Sect. 4 , and in Sect. 5 we summarize our conclusions. 


\section{Computational methods}

\subsection{Zooming coordinates and basic equations}

For any dependent function $f$ of radius and time, a generalized self-similar solution is characterized by the following decomposition (Munier \& Feix 1983):

$f(r, t)=g(t) \cdot f^{\prime}\left(r^{\prime}, t^{\prime}\right)$,

where $f^{\prime}$ is allowed to depend both on a rescaled radius $r^{\prime}=\xi(t) r$ and time $t^{\prime}=\nu(t)$. The time dependence of the function $f^{\prime}$ permits to study the convergence of the evolution toward self-similar solutions, which are obtained when $t^{\prime}$ asymptotically disappears from $f^{\prime}$. Based on these relations, Bouquet et al. (1985) first derived the explicit forms of the scaling functions $g(t), \xi(t)$, and $\nu(t)$ for the self-similar collapse of a polytropic gaseous sphere. With these transformations, the collapse of the unperturbed sphere in the ordinary $(r, t)$-space corresponds to a stationary solution in the rescaled $\left(r^{\prime}, t^{\prime}\right)$-space. Using the same transformations, Blottiau et al. (1988) derived self-similar solutions for the gravitational collapse of polytropic spheres, corresponding to the plateau - outer envelope solution observed in various numerical calculations. More recently, Matsumoto et al. (1997) and Matsumoto \& Hanawa (1999) wrote down the form of the self-similar transformations for the case of isothermal flows, and performed hydrodynamical calculations in the rescaled space to study the formation of self-similarly collapsing disks and bars during the contraction of a rotating gas cloud. They called the rescaled position $\boldsymbol{r}^{\prime}$ and time $t^{\prime}$ zooming coordinates because calculating the evolution in the transformed $\left(r^{\prime}, t^{\prime}\right)$-space allows the collapse to be followed up to very high densities while maintaining sufficient resolution in the ordinary space.

In spherical polar coordinates $(r, \theta, \phi)$, the transformation to the zooming coordinates for isothermal flows is given by

$\boldsymbol{r}^{\prime}=\left(r^{\prime}, \theta^{\prime}, \phi^{\prime}\right)=\left[\frac{r}{c_{\mathrm{S}}\left(t_{0}-t\right)}, \theta, \phi\right]$,

$t^{\prime}=-\ln \left[\frac{\left(t_{0}-t\right)}{t_{\mathrm{ff}}}\right]$,

where $c_{\mathrm{s}}$ is the isothermal speed of sound, $t_{0}$ is a reference time, and $t_{\mathrm{ff}}=\left(3 \pi / 32 G \rho_{0}\right)^{1 / 2}$ is the free-fall time for collapse, where $G$ is the gravitational constant and $\rho_{0}$ is a reference density. Differentiating $t^{\prime}$ with respect to $t$ in Eq. (3) yields the relation

$\mathrm{d} t^{\prime}=\frac{\mathrm{d} t}{\left(t_{0}-t\right)}$.

According to the transformations (2) and (4), the radial length $r$ and the time scale $\mathrm{d} t$ shorten as the time $t \rightarrow t_{0}$ for fixed $r^{\prime}$ and $\mathrm{d} t^{\prime}$.

In the transformed zooming space, the hydrodynamic equations describing the gravitational collapse of an isothermal rotating gas cloud may be written as

$\frac{\partial \rho^{\prime}}{\partial t^{\prime}}+\nabla^{\prime} \cdot\left(\rho^{\prime} \boldsymbol{v}^{\prime}\right)=\rho^{\prime}$

$$
\frac{\partial\left(\rho^{\prime} \boldsymbol{v}^{\prime}\right)}{\partial t^{\prime}}+\nabla^{\prime} \cdot\left(\rho^{\prime} \boldsymbol{v}^{\prime} \otimes \boldsymbol{v}^{\prime}\right)+\nabla^{\prime} \rho^{\prime}+\rho^{\prime} \nabla^{\prime} \Phi^{\prime}=2 \rho^{\prime} \boldsymbol{v}^{\prime}
$$

$\nabla^{\prime 2} \Phi^{\prime}=\rho^{\prime}$

where $\nabla^{\prime}$ denotes the gradient operator in the zooming space. The mass density $\rho$, the Eulerian fluid velocity $\boldsymbol{v}=$ $\left(v_{r}, v_{\theta}, v_{\phi}\right)$, and the gravitational potential $\Phi$ transform according to the following relations

$\rho^{\prime}=4 \pi G\left(t_{0}-t\right)^{2} \rho$,

$\boldsymbol{v}^{\prime}=\left(v_{r}^{\prime}, v_{\theta}^{\prime}, v_{\phi}^{\prime}\right)=\left[\frac{v_{r}}{c_{\mathrm{S}}}+r^{\prime}, \frac{v_{\theta}}{c_{\mathrm{s}}}, \frac{v_{\phi}}{c_{\mathrm{S}}}\right]$,

$\Phi^{\prime}=c_{\mathrm{S}}^{-2} \Phi$,

respectively. The $\phi$-component of the momentum Eq. (6) is written in terms of the specific angular momentum $A=$ $r^{2} \sin \theta v_{\phi}$, which transforms as

$A^{\prime}=\frac{A}{c_{\mathrm{s}}^{2}\left(t_{0}-t\right)}$.

Note that all primed quantities as defined in the zooming space are dimensionless. Compared to their counterparts in the ordinary space, the equations of continuity (5) and momentum (6) are almost invariant under the transformation, except for the source term on the right-hand side, whereas the Poisson Eq. (7) retains essentially the same form, except for the multiplicative factor $4 \pi G$ on the density. The existence of a mass source term in Eq. (5) shows that stationary flows might exist for which all primed functions become independent of $t^{\prime}$. In addition, the source term appearing in the Euler equation is proportional to the velocity and so it may be regarded as a friction term. It can effectively drive a system asymptotically to a stationary equilibrium.

\subsection{Numerical techniques}

According to relations (2) and (4), the radial length $\Delta r$ and the time interval $\Delta t$ as measured in the ordinary space are proportional to $t_{0}-t$. For fixed $\Delta r^{\prime}$ and $\Delta t^{\prime}$, this allows the numerical resolution to change adaptively in accordance with the density increase since $\rho \propto\left(t_{0}-t\right)^{-2}$. Evidently, this method involves solving the governing equations in the zooming space rather than in the ordinary one. Here Eqs. (5)-(7) are solved numerically using a modified version of the spherical-coordinate code described in full by Sigalotti (1998b). Because of the quasi-invariance of Eqs. (5)-(7), the same code can be used with only minor changes.

As in the original version, the code employs a multistep solution procedure in which the source and convective terms are evaluated separately. This is done through the use of spatially second-order accurate finite differences. Temporal second-order is also achieved by means of a predictor-corrector treatment of both the convective and source hydrodynamical terms. Advection of mass is performed using a generalization to spherical coordinates of 
the van Leer monotonic interpolation method. Consistent advection is enforced by calculating the mass fluxes $\rho^{\prime} \boldsymbol{v}^{\prime}$ at the cell surfaces as the product of a monotonically interpolated density by a linearly interpolated velocity. For the calculations of this paper, however, the momentum fluxes $\rho^{\prime} \boldsymbol{v}^{\prime} \otimes \boldsymbol{v}^{\prime}$ were determined by multiplying the mass fluxes with a monotonically interpolated velocity at the cell surfaces. This small change results in increased numerical diffusion thereby allowing the use of larger timesteps while maintaining numerical stability. The amount of diffusion implied by this change has relatively minor effects on the flow near sharp discontinuities. This has been tested using the one-dimensional version of the code through Noh's (1987) problem for the generation and propagation of a spherical shock wave out from the origin $(r=0)$. The resulting density, velocity, pressure and specific internal energy profiles are as expected for a second-order accurate code, and compared with the less diffusive form of the code the profiles as well as the instantaneous position of the shock are essentially unaltered, except for an effective damping of spurious post-shock oscillations arising near the discontinuity in the density, pressure and velocity. As in the original code, taking the difference replacements for the convective terms in Eqs. (5) and (6) and summing over the zooming grid, the fluxes in each coordinate direction will cancel out in pairs such that the time rate of change of the numerically advected quantities will only depend on the flux across the outer boundary. This ensures global conservation of mass and momentum in the solution when transforming it to the ordinary space. The source terms on the right-hand side of Eqs. (5) and (6) are evaluated separately from the other contributions using the following scheme

$\rho^{\prime a}=\rho^{\prime n} \cdot \exp \left(\Delta t^{\prime}\right)$

$\boldsymbol{v}^{\prime a}=\boldsymbol{v}^{\prime n} \cdot \exp \left(2 \Delta t^{\prime}\right)$

where $\rho^{\prime n}$ represents a cellwise density value at the beginning of the timestep and $\boldsymbol{v}^{\prime n}$ is the cellwise velocity vector after evaluation of the gravitational and pressure forces.

The Poisson equation is solved by a spherical harmonic expansion, including terms up to $l, m=16$. This procedure allows for a separation of the radial and angular variables such that the three-dimensional Eq. (7) is replaced by a set of ordinary differential equations for the amplitudes $\Phi_{l, m}^{\prime}\left(r^{\prime}\right)$, which are efficiently solved by means of a tridiagonal matrix algorithm. The $r^{\prime}$-grid is uniformly spaced and kept so during the whole calculation. A radial resolution consisting of $1+n_{r^{\prime}}=51,101$, and 201 (including the origin $r^{\prime}=0$ ) has been used for the calculations of this paper. The $\theta^{\prime}$-grid consists of $n_{\theta^{\prime}}=34$ fairly equidistant points for $0 \leq \theta^{\prime} \leq \pi / 2$ in all models, with symmetry through the cloud's equatorial plane being used to represent the bottom hemisphere of the cloud. The $\phi^{\prime}$-grid is also uniformly spaced with $n_{\phi^{\prime}}=64$ for $0 \leq \phi^{\prime}<\pi$, corresponding to $n_{\phi^{\prime}}=128$ effective points due to the assumption of $\pi$-symmetry. Thus, in a Fourier decomposition $\left(\mathrm{e}^{i m \phi}\right)$ only even modes can grow. Preliminary test calculations for the Gaussian cloud model (described in Sect. 3) by retaining terms up to $l, m=16$ and 32 in the spherical harmonic expansions have produced almost undistinguishable results implying that reasonable accuracy is achieved by including terms up to $l, m=16$. Truncating the expansions in this way is equivalent to filtering the solution against higher-order perturbation modes. Since for the present models the amplitudes of the $m>16$ modes always keep down at a level less than $\sim 10^{-2}$ even late in the collapse, their contribution remains small and so their exclusion does not represent a significant source of error. At the external surface of the zooming grid we impose an outflow boundary condition such that no disturbances can propagate into the domain of calculation from the surface. This boundary condition is naturally implemented because from Eq. (9), it follows that the gas flows out supersonically from the domain of calculation. The supersonic outflow is due to the apparent motion with respect to the zooming coordinates. The models with 51 radial points took about $1.0 \times 10^{5}$ timesteps to complete the evolution, corresponding to about $35 \mathrm{hr}$ of CPU time on a single Origin 2000 processor. The high-resolution models with 101 and 201 radial points required about $2.0 \times 10^{5}$ and $6.0 \times 10^{5}$ timesteps, respectively, corresponding to about 6 and 42 days of CPU time on an IBM Power 3 processor.

\section{A comparison test calculation}

In this section we present the results of a protostellar collapse test model with initial conditions corresponding to a centrally condensed, rapidly rotating cloud of $1 M_{\odot}$ and radius $R=5.0 \times 10^{16} \mathrm{~cm}(\approx 0.016 \mathrm{pc})$. The central condensation is obtained assuming a Gaussian density profile given by $\rho(r)=\rho_{0} \exp \left[-(r / b)^{2}\right]$, where $\rho_{0}=$ $1.7 \times 10^{-17} \mathrm{~g} \mathrm{~cm}^{-3}$ is the central density and $b \approx 0.577 R$ is a length chosen such that the central density is 20 times the value at the boundary surface. This spherically symmetric density distribution is perturbed with a small amplitude $(a=0.1), m=2$ density variation of the form $\rho(r, \phi)=\rho(r)[1+a \cos (m \phi)]$. The model has ideal gas thermodynamics at a fixed temperature of $10 \mathrm{~K}$ and a chemical composition of $X=0.769, Y=0.214$, and $Z=0.017$, implying a mean molecular weight $\mu \approx 2.28$. With these parameters, the initial ratio of the thermal to the absolute value of the gravitational energy is $\alpha \approx 0.265$. Uniform rotation at a rate of $\omega=1.0 \times 10^{-12} \mathrm{~s}^{-1}$ is assumed, corresponding to an initial ratio of the rotational to the self-gravitational energy of $\beta \approx 0.16$. The isothermal approximation is valid for densities roughly in the range of $\sim 10^{-19}$ to $\sim 10^{-13} \mathrm{~g} \mathrm{~cm}^{-3}$. The collapse of the Gaussian cloud was assumed to remain isothermal beyond densities of $\sim 10^{-13} \mathrm{~g} \mathrm{~cm}^{-3}$ just to check the ability of the code to reproduce the filamentary solution found by Truelove et al. (1997) and Boss et al. (2000) working at very high spatial resolution.

Truelove et al. (1997) found that in isothermal collapse calculations artificial fragmentation is avoided if the mass within a Cartesian cell never exceeds $1 / 64$ of the Jeans mass $\rho \lambda_{\mathrm{J}}^{3}$, where $\lambda_{\mathrm{J}}=\left(\pi c_{\mathrm{S}}^{2} / \rho G\right)^{1 / 2}$ denotes 
the Jeans length. Previous low-resolution calculations for the Gaussian model not adhering to the Jeans condition produced a quadruple protostellar core by the end of the isothermal collapse (Boss 1991, 1993; Klapp et al. 1993). Evidently, these calculations underwent artificial fragmentation as a result of the growth of numerical noise caused by insufficient spatial resolution. Starting with low initial resolution $\left(n_{r}=50, n_{\phi}=64\right)$, Boss (1998) recalculated the collapse of the Gaussian cloud by redefining the radial grid in such a way as to satisfy the Jeans mass condition

$$
\begin{aligned}
\Delta x & =\left(\Delta x_{r} \Delta x_{\theta} \Delta x_{\phi}\right)^{1 / 3} \\
& =\left(r^{2} \sin \theta \Delta r \Delta \theta \Delta \phi\right)^{1 / 3}<\frac{\lambda_{J}}{4},
\end{aligned}
$$

and the three Jeans length conditions

$$
\begin{aligned}
& \Delta x_{r}=\Delta r<\frac{\lambda_{\mathrm{J}}}{4}, \\
& \Delta x_{\theta}=r \Delta \theta<\frac{\lambda_{\mathrm{J}}}{4}, \\
& \Delta x_{\phi}=r \sin \theta \Delta \phi<\frac{\lambda_{\mathrm{J}}}{4},
\end{aligned}
$$

for spherical coordinates, and the cloud was still found to fragment artificially into a well-defined binary system. Only when the spatial resolution was increased to $n_{r}=$ $200, n_{\phi}=128$, the solution changed behavior (Boss et al. 2000). That is, instead of fragmenting into a binary, the cloud collapsed to form a central bar, which thereafter condensed into a thin filament in agreement with the AMR results of Truelove et al. (1997). Thus, it appears that for spherical grids, the four Jeans constraints (12) and (13) are necessary but not sufficient for realistic fragmentation, and that sufficiently high spatial resolution is also required to ensure a converged solution.

Here we provide a further intercomparison test for the isothermal Gaussian cloud using a completely different numerical framework compared to those employed by Truelove et al. (1997) and Boss et al. (2000). Since the collapse is entirely calculated in the zooming coordinate grid, the radial cell spacings as measured in the ordinary space shrink in proportion to $t_{0}-t$. Note that when $t_{0}$ is smaller, the central cloud region is zoomed with higher magnification. For this test, we use $1+n_{r^{\prime}}=101$ radial points and a value of $t_{0}=1.40 t_{\mathrm{ff}}$, which is enough to resolve the Jeans length through 7 orders of magnitude increase in density. During the first free-fall time, the overall cloud flattens about its equatorial plane to form a rotationally supported disk. In this phase, the gas flows outward in the zooming coordinates, corresponding to a general mild infall in the ordinary space. After about 1.28 $t_{\mathrm{ff}}$, the central cloud regions experience more rapid collapse and, as a consequence, the central density increases. By $1.32 t_{\text {ff }}$, when $\rho_{\max } \approx 1.38 \times 10^{3} \rho_{0}$, the innermost regions start deforming into an elongated bar as a result of the growth of a non-axisymmetric $m=2$ instability. Figures $1 \mathrm{a}$ and $\mathrm{b}$ display equatorial density contours of the central cloud in the ordinary space at $1.358 t_{\mathrm{ff}}$, when the bar is already evident. Matsumoto \& Hanawa (1999) studied the mechanisms responsible for bar formation, and found that growth of the shear component of the velocity may induce the deformation of a non-uniformly rotating disk into a barlike configuration. During the bar formation stage, the zooming radial velocity $v_{r}^{\prime}=v_{r} / c+r^{\prime}$ inside the disk decreases as $v_{r} / c_{\mathrm{S}}=-\left|v_{r}\right| / c_{\mathrm{S}}$ increases in magnitude compared to $r^{\prime}$. As the central collapse continues to even higher densities, these terms roughly balance making $v_{r}^{\prime} \approx 0$. When this occurs, the centralmost cloud regions approach a rotating steady state in the zooming space. This corresponds to a self-similar solution in the ordinary space. In fact, subsequent collapse of the central bar proceeds self-similarly. That is, the bar shrinks faster in the direction of the cylindrical radius than along the long axis. As a consequence, the bar becomes progressively narrower and a singular filament forms as shown in Fig. 1c at 1.36 $t_{\mathrm{ff}}$. At this time, the filament has a maximum density of $\sim 1.01 \times 10^{6} \rho_{0}$. Because of the assumption of isothermality, it will continue undergoing indefinite collapse upon itself. Figure $1 \mathrm{~d}$ depicts the filament at $1.361 t_{\mathrm{ff}}$ by the end of the calculation, when $\rho_{\max } \sim 1.01 \times 10^{7} \rho_{0}$. The details of the collapse of the filament are clarified in Fig. 2, which shows the velocity field in the equatorial plane at the same time of Fig. 1d. We see that mass with relatively high angular momentum accretes the filament at its ends, while collapse of low angular momentum mass occurs preferentially toward its long axis.

The filament shown in Figs. 1c and d strongly resembles the self-similar solution first found by Inutsuka \& Miyama (1992). They predicted by linear analysis that as long as the mass per unit length of an infinitely long cylinder is much greater than the equilibrium value $(M / L)_{\mathrm{e}}=2 c_{\mathrm{s}}^{2} / G$, fragmentation of the cylinder will not occur as long as the collapse will remain isothermal. By the time of Fig. 1c, the filament has $(M / L) \approx 1.27 \times$ $10^{16} \mathrm{~g} \mathrm{~cm}^{-1}$, which is greater than the equilibrium value of $1.09 \times 10^{16} \mathrm{~g} \mathrm{~cm}^{-1}$. Thus, we expect the filament to collapse isothermally toward a singularity without fragmenting. Figure 3 describes the evolution of the four grid sizes and the Jeans length during the cloud collapse. Evidently, satisfaction of the Jeans constraints (12) and (13) combined with high resolution allowed us to follow the isothermal collapse up to the point where filament formation is expected. This result is in agreement with the AMR code calculation of Truelove et al. (1997) and the refined spherical code calculation of Boss et al. (2000). The agreement between these three independent codes, each working at very high spatial resolution, implies once more that the filamentary solution is the correct one. Although the outcome of the isothermal collapse of the Gaussian cloud is shown to be a singular filament, a physically realistic solution would require the inclusion of non-isothermal heating. Following this line, Bate \& Burkert (1997) performed SPH calculations of the isothermal collapse of initially uniform-density clouds by fulfilling the Jeans resolution conditions. They predicted the formation of a long bar which thereafter fragmented into four clumps as a result of allowing heating of the central cloud regions to 

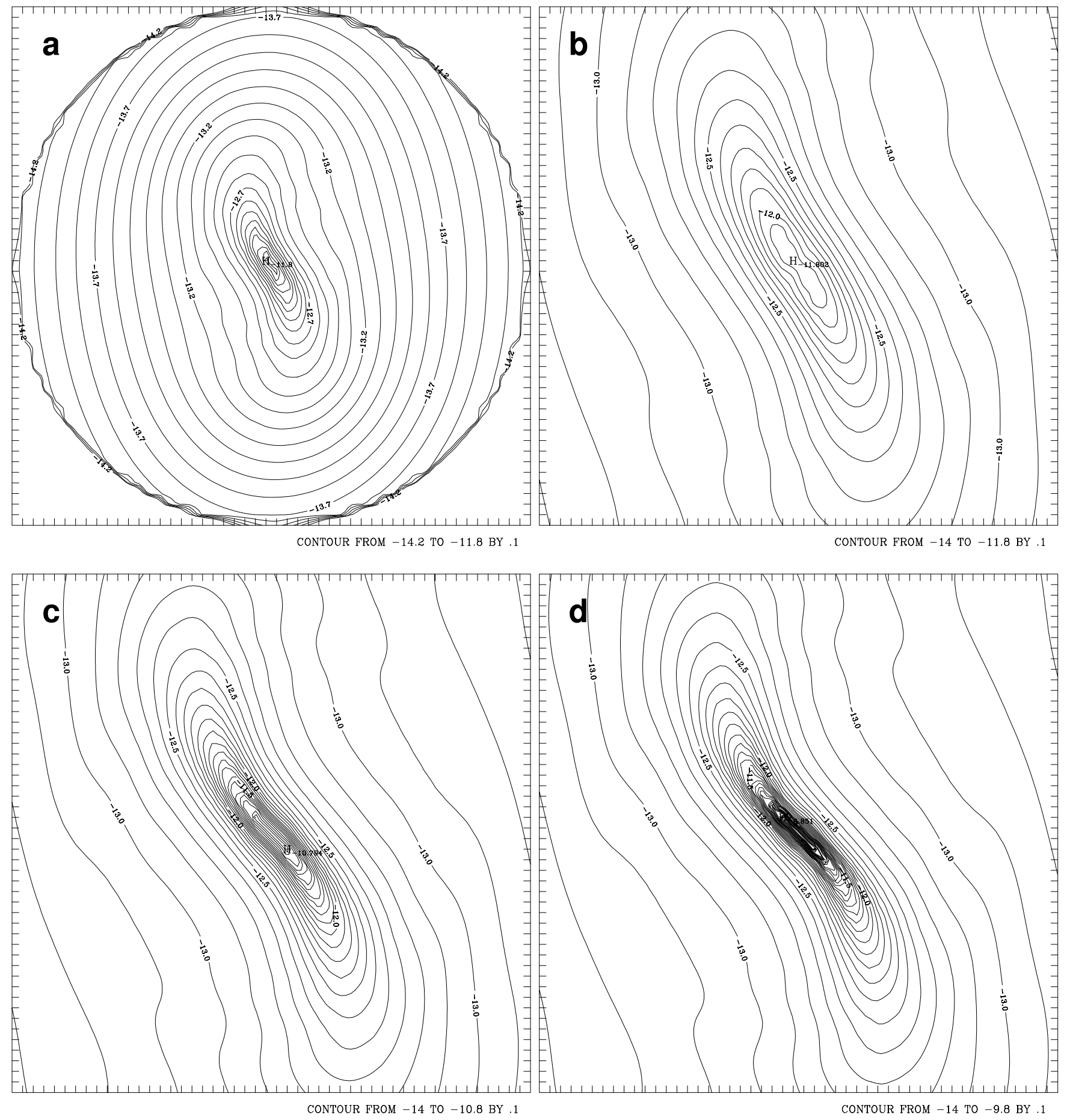

Fig. 1. Density contours in the equatorial plane for the isothermal Gaussian cloud at three different times: a) and b) $1.358 t_{\mathrm{ff}}$, c) $1.360 t_{\mathrm{ff}}$, and d) $1.361 t_{\mathrm{ff}}$. The contour lines correspond to the logarithm of the density. Maximum densities and box radii are: a) $9.43 \times 10^{4} \rho_{0}, 1.5 \times 10^{15} \mathrm{~cm}$. b) Same as a), but with box radius $=6.0 \times 10^{14} \mathrm{~cm}$. c) $1.01 \times 10^{6} \rho_{0}, 5.0 \times 10^{14} \mathrm{~cm}$. d) $1.01 \times 10^{7} \rho_{0}, 5.0 \times 10^{14} \mathrm{~cm}$. Formation of an intermediate barlike central core is evident in a) and b), while formation of a dense filamentary structure is clearly shown in $\mathbf{c}$ ) and $\mathbf{d}$ ). In each plot the density is expressed by its values in the ordinary coordinates.

guarantee preservation of the Jeans mass resolution beyond densities of $\approx 10^{-13} \mathrm{~g} \mathrm{~cm}^{-3}$ in their calculations. Similarly, Klein et al. (1998) reported AMR calculations for the same model of Bate \& Burkert (1997), but this time using an equation of state which makes the tran- sition from the isothermal to the non-isothermal regime in a smooth fashion. They also found a forming long bar which, after becoming optically thick, fragmented into a binary core system. Thus the presence of gradual heating in the densest cloud regions increases the gas pressure 


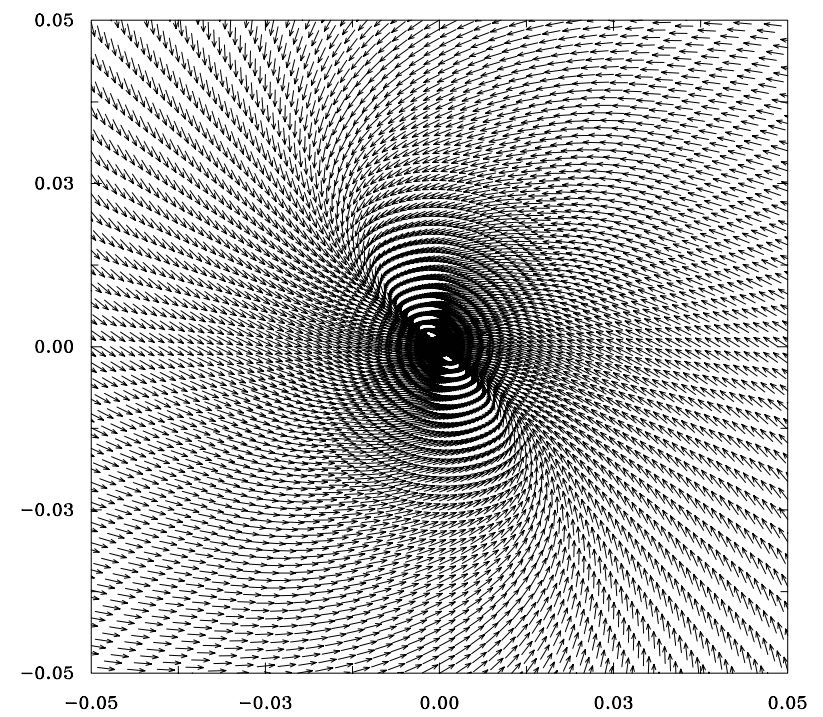

Fig. 2. Velocity vectors in the equatorial plane for the isothermal Gaussian cloud at the same time of Fig. 1d. A radius of $5.0 \times 10^{14} \mathrm{~cm}$ is shown. The maximum velocity is $0.685 \mathrm{~km} \mathrm{~s}^{-1}$. The velocities are expressed by their values in the ordinary coordinates.

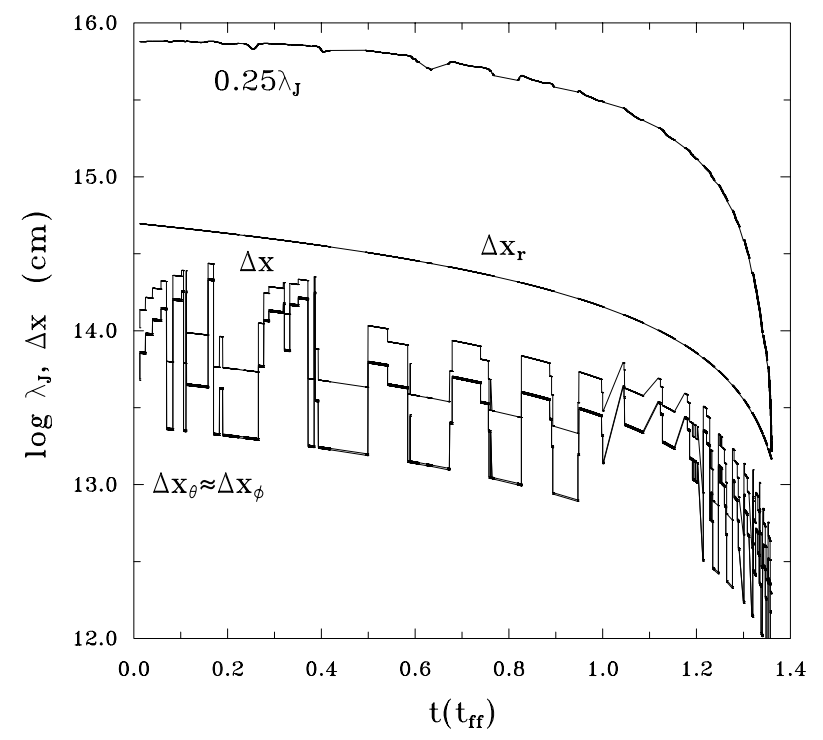

Fig. 3. Dependence of the four spherical grid sizes and the Jeans length on evolution time, evaluated at the location of the maximum density in the equatorial plane, for the isothermal Gaussian cloud. The angular resolution used is such that $\Delta x_{\theta} \approx \Delta x_{\phi}$ at the midplane.

forces with a consequent retardation of the collapse, allowing fragmentation into a protostellar binary system to occur at the same maximum density where the isothermal collapse yields a thin filament (Boss et al. 2000). This implies that fragmentation appears to depend sensitively on both the numerical resolution and the detailed thermodynamical treatment.
Table 1. Prolate isothermal cloud cores with $\alpha \approx 0.22$.

\begin{tabular}{lcccccc}
\hline Model & $\begin{array}{c}\omega_{0} \\
\left(\mathrm{rad} \mathrm{s}^{-1}\right)\end{array}$ & $\beta$ & $\begin{array}{c}t_{0} \\
\left(t_{\mathrm{ff}}\right)\end{array}$ & $\frac{(M / L)}{(M / L)_{\mathrm{e}}}$ & $\frac{L}{\lambda_{\mathrm{J}}}$ & $\begin{array}{c}\text { Time } \\
\left(t_{\mathrm{ff}}\right)\end{array}$ \\
\hline P1 & $1.0(-13)$ & 0.246 & 1.42 & 2.1 & 10 & 1.397 \\
P2 & $7.5(-14)$ & 0.138 & 1.30 & 3.9 & 17 & 1.265 \\
P3 & $3.2(-14)$ & 0.025 & 1.18 & 4.0 & 19 & 1.145 \\
P4 & $1.0(-14)$ & 0.0025 & 1.18 & 4.8 & 21 & 1.123 \\
P5 & $3.2(-15)$ & 0.00025 & 1.18 & 4.7 & 25 & 1.121 \\
\hline
\end{tabular}

\section{Collapse of prolate cloud cores}

This section describes the results of five isothermal models of prolate cloud core collapse, with initial parameters as listed in Table 1 . In all cases, the starting point of collapse is taken to be a centrally condensed configuration, with the central density $\rho_{0}$ being a factor of 20 higher than the boundary density. In addition, the threedimensional shape of the initial configuration is chosen to resemble the prolate molecular cloud cores studied by Myers et al. (1991) and Ryden (1996). Such a density profile can be obtained by mapping onto the computational grid a Gaussian, prolate spheroidal density distribution of the form

$\rho(x, y, z)=\rho_{0} \cdot \exp \left(-\frac{x^{2}}{a^{2}}-\frac{y^{2}+z^{2}}{b^{2}}\right)[1+0.1 \operatorname{RAN}(\phi)],(14)$

where $\rho_{0}=1.0 \times 10^{-19} \mathrm{~g} \mathrm{~cm}^{-3}$ for all cases and $\operatorname{RAN}(\phi)$ is a random number in the interval $[0,1]$, added to introduce a noisy dependence with the $\phi$-coordinate. The values of $a \approx 1.16 R$ and $b \approx 0.58 R$ are chosen such that the prolate shapes correspond to axial ratios of $2: 1$, where $R=6.4 \times 10^{17} \mathrm{~cm}(\approx 0.21 \mathrm{pc})$ is the outer radius of the spherical grid. The gas is assumed to have a composition of $X=1, Y=Z=0$ (corresponding to pure molecular hydrogen) and a temperature of $10 \mathrm{~K}$. With these parameters, all five models have an initial $\alpha \approx 0.22$ and a nominal mass of $19.3 M_{\odot}$. The entries in Table 1 define a sequence of calculations differing only in the initial value of the uniform angular velocity. This produces values of $\beta$ in the range $0.25 \leq \beta \leq 0.00025$. Barranco \& Goodman (1998) measured values of $\beta$ well within this range in cores with detectable rotation.

Previous collapse calculations of prolate cloud models have shown that the density profile given by Eq. (14) is conducive to fragmentation (Boss 1993; Boss \& Myhill 1995; Sigalotti \& Klapp 1997; Sigalotti 1998a). In particular, the same initial conditions of models P1 and P3-P5 were previously used by Boss (1993) (see his models I1-I4). He performed low-resolution calculations not satisfying the Jeans conditions (12) and (13), finding that in all cases the clouds collapsed to form intermediate narrow cylinders that subsequently fragmented into binary systems with separations on the order of 300-2000 AU. Here we recalculate the same models employing sufficiently high 
spatial resolution and satisfying the Jeans conditions in order to assess whether they will still fragment during their isothermal collapse or condense all the way toward a singular filament without fragmenting, as predicted by the linear analysis of Inutsuka \& Miyama (1992). If the mass per unit length of a collapsing cylindrical cloud exceeds the equilibrium value $2 c_{\mathrm{s}}^{2} / G$, perturbations do not grow much and the cloud will undergo indefinite collapse toward its axis. In this case, fragmentation is not expected as long as the collapse remains isothermal. Subsequent evolution may, however, lead to fragmentation after or during a change in the equation of state. Recently, Boss et al. (2000) found using nonisothermal thermodynamics, with Eddington approximation radiative transfer and detailed equations of state, that nonisothermal heating may retard the collapse allowing fragmentation to occur in the Gaussian cloud model of Sect. 3 before the formation of a singular filament. If the predictions of Inutsuka \& Miyama (1992) are also applicable to collapsing isothermal prolate spheroids, we then expect them to produce singular filaments rather than binary systems. Under more realistic conditions, the collapse of such prolate clouds may eventually lead to fragmentation due to thermal retardation rather than to their elongated geometry as was often suggested by all previous studies.

Models P1-P5 were first calculated using a radial grid consisting of $1+n_{r^{\prime}}=51$ uniformly distributed points, and then recalculated by increasing the radial resolution to $1+n_{r^{\prime}}=201$ equidistant points. In spite of the adaptive nature of the zooming coordinates, a resolution of $1+n_{r^{\prime}} \geq 201$ radial points is necessary to guarantee satisfaction of the Jeans conditions for these models when densities beyond the limit of validity of the isothermal regime are reached. The higher resolution models all collapsed in a similar fashion, producing singular filaments which did not fragment during the isothermal phase of collapse. Figure 4 shows equatorial density contours at four distinct times for the evolution of model P1 with $1+n_{r^{\prime}}=201$ points. The cloud structure at the beginning of collapse is shown in Fig. 4 a, when $\rho_{\max } \approx 1.1 \rho_{0}$. During the first $1.35 t_{\mathrm{ff}}$, the maximum density grows very slowly and the overall cloud flattens about its equatorial plane to form a rotationally supported disk. After the disk formation phase, the central infall proceeds primarily toward the major axis of the cloud, making the central core more prolate as it collapses. Figure $4 \mathrm{~b}$ shows the zoomed central parts of the cloud at $1.356 t_{\mathrm{ff}}$, when $\rho_{\max } \approx 1.25 \times 10^{3} \rho_{0}$. After this time, the maximum density grows at a much faster rate and the central regions condense self-similarly into a thin bar as shown in Fig. 4c at $1.395 t_{\mathrm{ff}}$, when $\rho_{\max } \approx 1.40 \times 10^{5} \rho_{0}$. Subsequent collapse is characterized by the bar shrinking into a singular filament. Figure $4 d$ shows the central filament at $1.396 t_{\mathrm{ff}}$ by the end of the isothermal collapse when $\rho_{\max } \approx 1.08 \times 10^{6} \rho_{0}$. Because of the noisy dependence of the initial density distribution, the innermost filament looks a bit irregular in shape and has a rather non-uniform structure. This is shown in Fig. 4d, where we see the occurrence of two density maxima within the innermost contour. These mild condensations may act as true perturbations which may then amplify rapidly during further collapse, causing fragmentation of the filament along its length. However, at such high densities the Jeans length is still well resolved and the mass per unit length of the filament, defined as the region with $\rho \geq 3.0 \times 10^{5} \rho_{0}$, is found to exceed its equilibrium value $(M / L)_{\mathrm{e}}=2 c_{\mathrm{s}}^{2} / G$ (see Table 1 ). We therefore expect the filament to undergo further isothermal collapse without fragmenting. To assess this point, the calculation was continued farther in time until $\rho_{\max } \approx 5.0 \times 10^{6} \rho_{0}$ was reached. By this time, the two maxima have not amplified yet implying that the timescale for collapse is effectively shorter than the growth time of the perturbations. We cannot follow the further collapse in this calculation because soon after when $\rho_{\max } \approx 7.0 \times 10^{6} \rho_{0}$, the local Jeans length $\lambda_{\mathrm{J}} / 4 \sim \Delta r$ at the point of maximum density, making the filament susceptible to artificial fragmentation. Fragmentation into a well-defined binary system was seen when the calculation of model P1 was repeated using $1+n_{r^{\prime}}=51$ points. In this case, $\lambda_{\mathrm{J}} / 4<\Delta r$ at much lower densities than the maximum value in Fig. $4 \mathrm{~d}$ and, as a consequence, formation of a binary occurred in much the same way as in previous calculations not adhering to the Jeans condition (see below for a direct comparison between the $1+n_{r^{\prime}}=51$ and 201 calculations for models P2 and P3).

In the present models, the transition from a prolate ellipsoid to a long bar and then from a bar to a singular filament occurs self-similarly. That is, the collapse proceeds faster toward the cylindrical radius than along the long axis, causing the ellipsoid to shrink into a bar and then the bar into a long filament. Similar results were also found by Matsumoto \& Hanawa (1999) when studying the deformation of a circular disk into a non-axisymmetric bar during the collapse of a rotating gas cloud. The similarity is shown in Fig. 5 for the case of model P1, which shows the density and velocity profiles on the equatorial plane. Figures $5 \mathrm{a}$ and $\mathrm{b}$ both depict the density profile along the short and long axis of the cloud, respectively. Each density profile has a plateau at the center and an envelope approximated by an $r^{-2}$ power law. Because of the zooming coordinates, the central cloud regions are continually zoomed during collapse causing the outer envelope region to leave the domain of calculation. As the central density increases, the plateau shrinks in size and the envelope structure changes little. The curves from "a" to "d" in each figure correspond to the epoch during which the prolate ellipsoid condenses into a bar and curves "e" to "g" describe the collapse of the bar into a thin filament. In Fig. 5b, we see that at the time of bar formation the density profile changes discontinuously at the end of the bar owing to a shock wave. The shock strength increases as the bar shrinks with the shock moving inward. Note that the small oscillations present along the bar (curve e) never amplify during further collapse (curves $\mathrm{f}$ and $\mathrm{g}$ ). Similarity of the solution at this two different epochs is also shown by the radial and rotational velocities. Figures $5 \mathrm{c}$ and e show that they decrease 

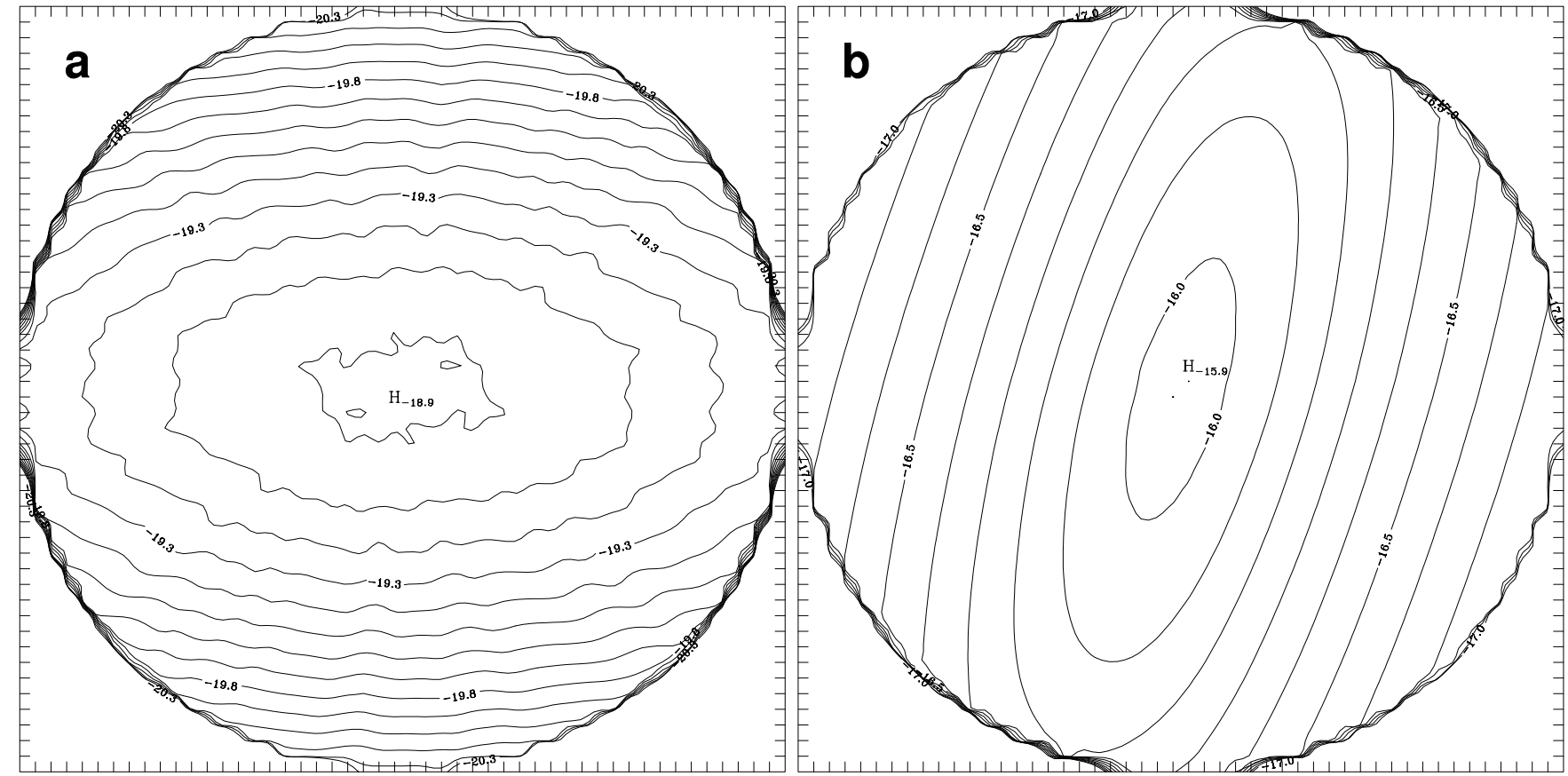

CONTOUR FROM -20.3 TO -19 BY .1

CONTOUR FROM -17 TO -16 BY .1
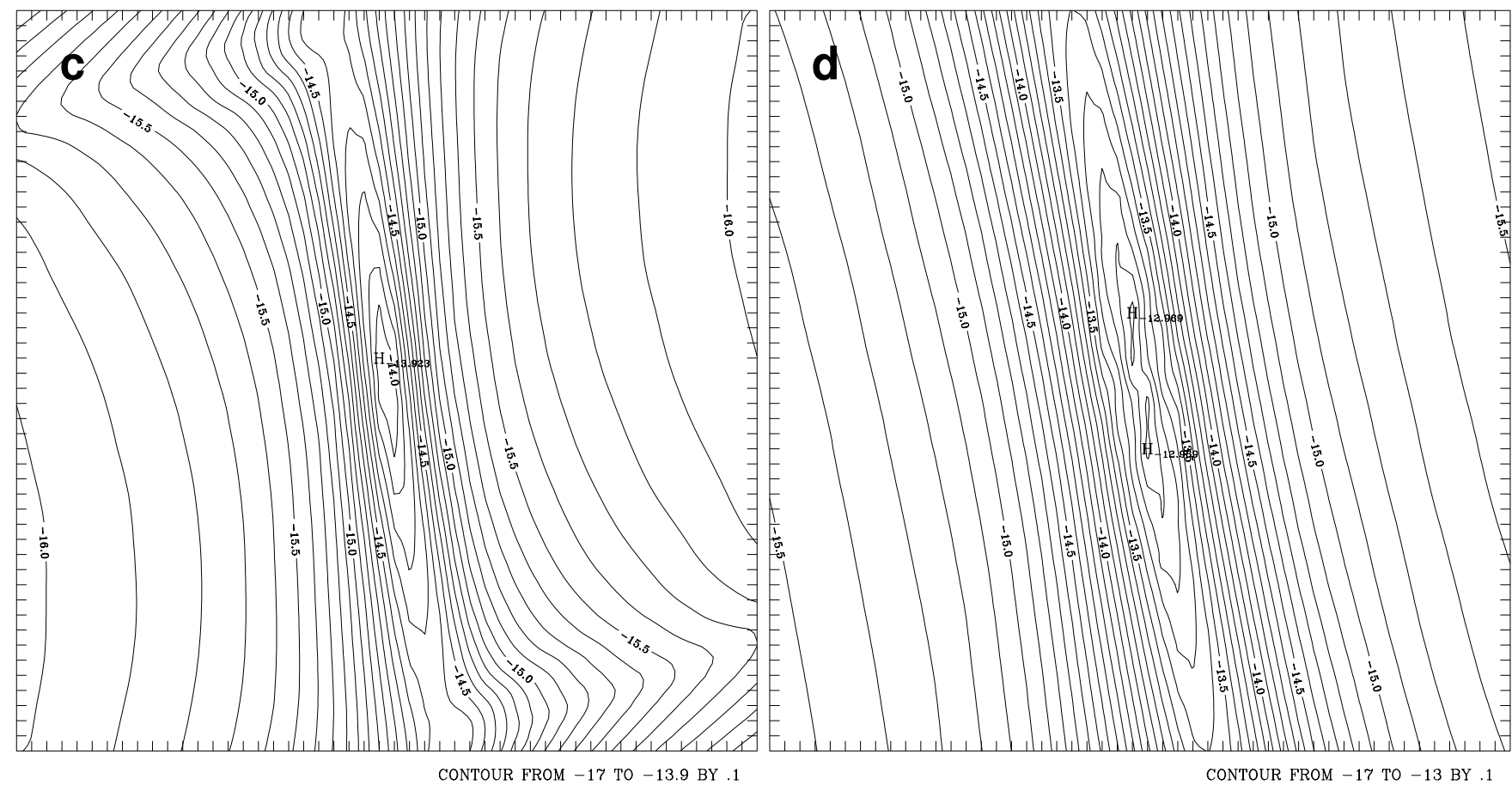

Fig. 4. Density contours in the equatorial plane for the evolution of model P1, with $1+n_{r^{\prime}}=201$ points, at four different times: a) $5.625 \times 10^{-3} t_{\mathrm{ff}}$, b) $1.356 t_{\mathrm{ff}}$, c) $1.395 t_{\mathrm{ff}}$, and d) $1.396 t_{\mathrm{ff}}$. The contour lines correspond to the logarithm of the density. Maximum densities and box radii are: a) $1.1 \rho_{0}, 6.3 \times 10^{17} \mathrm{~cm}$. b) $1.25 \times 10^{3} \rho_{0}, 2.8 \times 10^{16} \mathrm{~cm}$. c) $1.40 \times 10^{5} \rho_{0}, 8.0 \times 10^{15} \mathrm{~cm}$. d) $1.08 \times 10^{6} \rho_{0}, 2.0 \times 10^{15} \mathrm{~cm}$. The initial prolate spheroid is shown in a), the intermediate triaxial ellipsoid is shown in b), and the final filamentary core in $\mathbf{c}$ ) and $\mathbf{d}$ ). The magnification in d) implies a radial grid consisting of 10464 points covering the initial cloud radius.

proportionally to the radius in the region of the density plateau. Along the filament (Figs. 5d and f), the radial velocity also decreases with radius, while the rotational velocity is significantly greater toward the filament ends due to accretion of high angular momentum gas. Inside the filament region, the rotational velocity decreases rapidly toward the center consistent with accretion of low angular momentum mass occurring preferentially toward the long axis of the filament.

The change in cloud shape is quantified in Fig. 6 . The ordinate denotes the logarithm of the length of the three axes and the absissa denotes the logarithm of the 
L. Di G. Sigalotti and J. Klapp: Protostellar collapse models of prolate molecular cloud cores
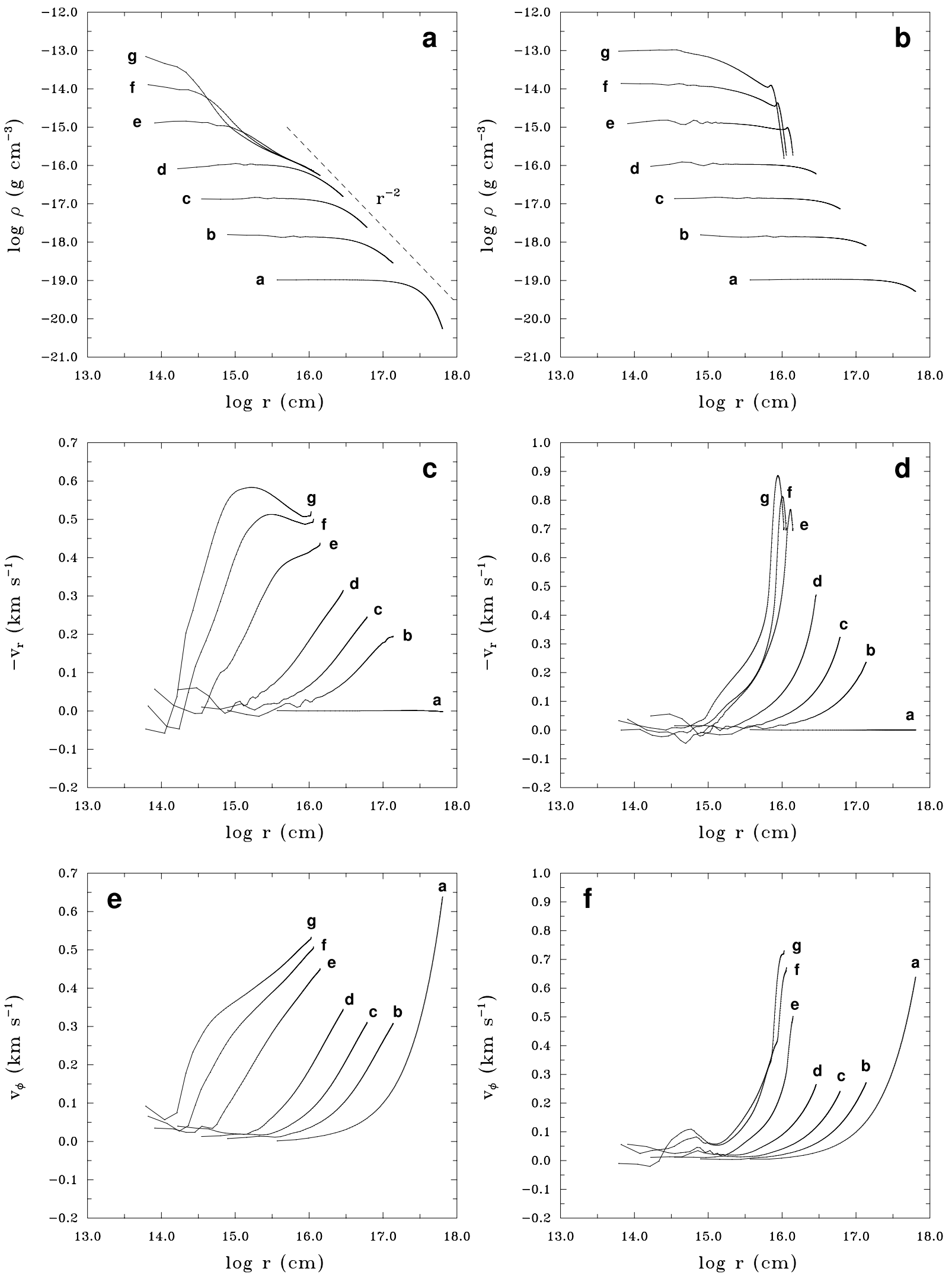

Fig. 5. Density, radial velocity, and rotational velocity profiles on the equatorial plane for model P1 at different times. Plots a) $-\mathbf{f})$ show the profiles in the direction perpendicular to the cloud's elongation and along the long axis, respectively. In each plot the times are: $t / t_{\mathrm{ff}}=5.625 \times 10^{-3}$ (curve a), 1.118 (curve b), 1.286 (curve c), 1.356 (curve d), 1.389 (curve e), 1.395 (curve f), and 1.396 (curve g). Similarity is shown in all of the variables. 


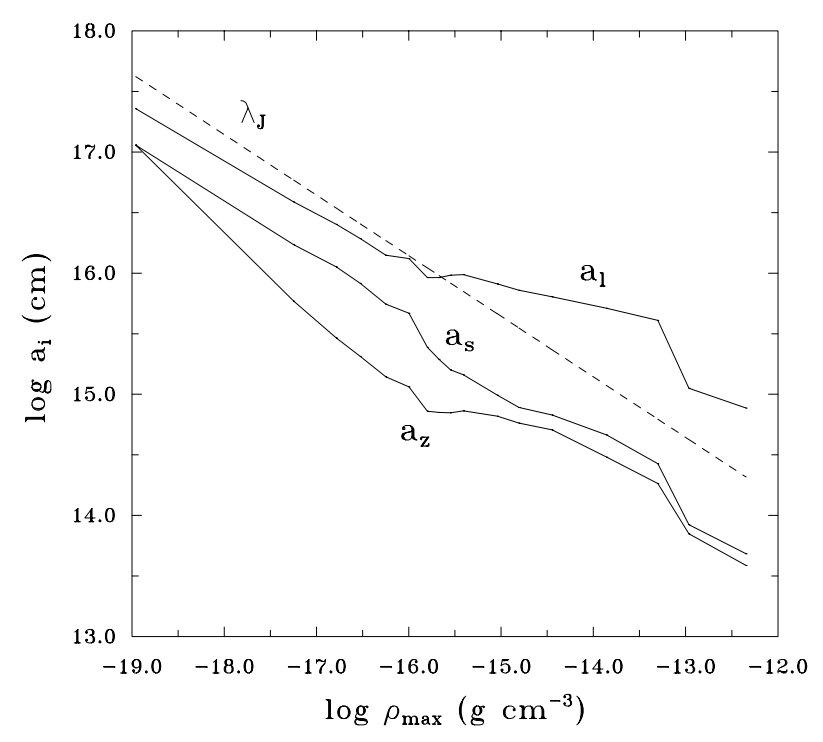

Fig. 6. Variation of the cloud's axis length in each of the three directions in the dense region for model P1. The symbol $a_{z}$ denotes the axis length in the direction of the rotation axis, while $a_{\mathrm{s}}$ and $a_{1}$ denote, respectively, the short and long axis in the $(x, y)$-plane. The dashed line gives the variation of the local Jeans length $\lambda_{\mathrm{J}}$ at the location of the maximum density.

maximum density. The axial lengths are calculated using the principal values of the moment of inertia

$I_{i j}=\int r_{i} r_{j} \rho(\boldsymbol{r}) \mathrm{d} V$,

and the total mass

$M=\int \rho(\boldsymbol{r}) \mathrm{d} V$,

where the integration is carried over the volume of the maximum elongated region that is guaranteed to be within the domain of calculation. The symbol $a_{z}$ denotes the axis length along the $z$-direction (rotation axis) and is defined by $\left(I_{z z} / M\right)^{1 / 2}$. Similarly, $a_{\mathrm{s}}$ and $a_{\mathrm{l}}$ denote the length of the short and long axes in the $(x, y)$-plane (equatorial plane), respectively. They are defined as $a_{\mathrm{s}}=\left(I_{y y} / M\right)^{1 / 2}$ and $a_{1}=\left(I_{x x} / M\right)^{1 / 2}$, where

$$
\begin{aligned}
I_{x x} & =\int \rho(r, \theta, \phi) r^{4} \sin ^{3} \theta \cos ^{2}\left(\phi-\phi_{0}\right) \mathrm{d} r \mathrm{~d} \theta \mathrm{d} \phi, \\
I_{y y} & =\int \rho(r, \theta, \phi) r^{4} \sin ^{3} \theta \sin ^{2}\left(\phi-\phi_{0}\right) \mathrm{d} r \mathrm{~d} \theta \mathrm{d} \phi, \\
I_{z z} & =\int \rho(r, \theta, \phi) r^{4} \sin ^{2} \theta \cos ^{2} \theta \mathrm{d} r \mathrm{~d} \theta \mathrm{d} \phi,
\end{aligned}
$$

where $\phi_{0}$ denotes the rotated azimuthal angle between the $x$-grid axis $(\phi=0)$ and the long axis of the cloud. At the beginning of collapse both $a_{z}$ and $a_{\mathrm{s}}$ coincides while $a_{1}=2 a_{z}=2 a_{\mathrm{s}}$. During the first phase of collapse, $a_{z}$ decreases more rapidly than the other two axes. This phase corresponds to the disk formation stage in which the cloud flattens about its equatorial plane. Note that during this phase $a_{1} \approx 2 a_{\mathrm{s}}$, and so the cloud preserves its initial axis ratio in the $(x, y)$-plane. Only when $\log \rho_{\max }>-16.0$, the central core becomes appreciably elongated as $a_{\mathrm{s}}$ decreases at a much faster rate and $a_{1}$ increases. This change in cloud shape occurs in the range $-16.0<\log \rho_{\max }<-15.0$, corresponding to the bar formation stage. Soon after $\log \rho_{\max } \approx-15.8$ is reached, $a_{z}$ expands and then decreases at a much slower rate compared to $a_{\mathrm{s}}$ until $a_{\mathrm{s}} \approx a_{z}$ during the stage of filament formation. For comparison, the dashed line shows the variation of the Jeans length at the location of the maximum density. At the beginning of the bar formation stage $a_{1} \sim \lambda_{\mathrm{J}}$ and thereafter becomes much longer than $\lambda_{\mathrm{J}}$. Thus, the forming filament is long enough to contain several times the Jeans length and therefore it may become unstable to fragmentation. The temporal increase of $a_{1}$ and the much rapid decrease of $a_{\mathrm{s}}$ around $\log \rho_{\max } \approx-16.0$ is due to the much stronger infall that sets in toward the long axis of the cloud.

As the amount of initial rotational energy $\beta$ is lowered in the sequence of models P1-P5, the timescale of filament formation shortens. As the centrifugal forces become less important, the collapse toward the major axis of the cloud occurs at a much faster rate than along the long axis; the evolution resulting in longer filaments by the end of the isothermal collapse. Figure 7 gives details of the equatorial density distribution later in the collapse of model P2 for both the high $\left(1+n_{r^{\prime}}=201\right)$ and low $\left(1+n_{r^{\prime}}=51\right)$ resolution calculations. Figures $7 \mathrm{a}$ and $\mathrm{b}$ shows the intermediate long bar at $1.261 t_{\mathrm{ff}}$, when $\rho_{\max } \approx 5.4 \times 10^{4} \rho_{0}$, and the final filament at $1.265 t_{\mathrm{ff}}$, when $\rho_{\max } \approx 1.7 \times 10^{6} \rho_{0}$, for the high-resolution case. For comparison, Figs. $7 \mathrm{c}$ and d show the results obtained working at much lower spatial resolution at $1.263 t_{\mathrm{ff}}$ and $1.269 t_{\mathrm{ff}}$, respectively. We see that at the same maximum density where a narrow bar forms in Fig. 7a, fragmentation into two pieces has already occurred in the lower resolution calculation (Fig. 7c). The fragments then grow in density producing a well-defined binary system (Fig. 7d) at a value of the maximum density comparable to that where a long filament forms in the $1+n_{\mathrm{r}^{\prime}}=201$ calculation. By the time of Fig. $7 \mathrm{c}$, the Jeans length $\lambda_{\mathrm{J}} / 4 \ll \Delta r$ at the location of the maximum density and so the model suffered from artificial fragmentation. In all low-resolution models, fragmentation of the bar begins when $\Delta r \sim \lambda_{\mathrm{J}} / 4$. As long as this happens, the maximum amplitude of the $m=2$ mode starts growing much faster than the bar can actually collapse upon itself. When $\Delta r \sim 10 \lambda_{\mathrm{J}} / 4$, the fragmentation process becomes evident with the bar clearly separating into two growing clumps as shown in Fig. 7c. As the clumps condense even more, the amplitudes of the $m=2$ mode experience rapid growth to a saturation value of $\sim 10^{2}$, while the local Jeans length decreases reaching values of $\sim 10^{-4} \Delta r$ or even less (Fig. 7d). This result strongly resembles those reported in previous studies of prolate cloud core collapse, implying that the reliability of the fragmentation observed in those models is really being affected by a violation of the Jeans condition. The present results indicate that as long as the Jeans length is resolved, the solution for the isothermal collapse of a prolate cloud core may change from a binary 


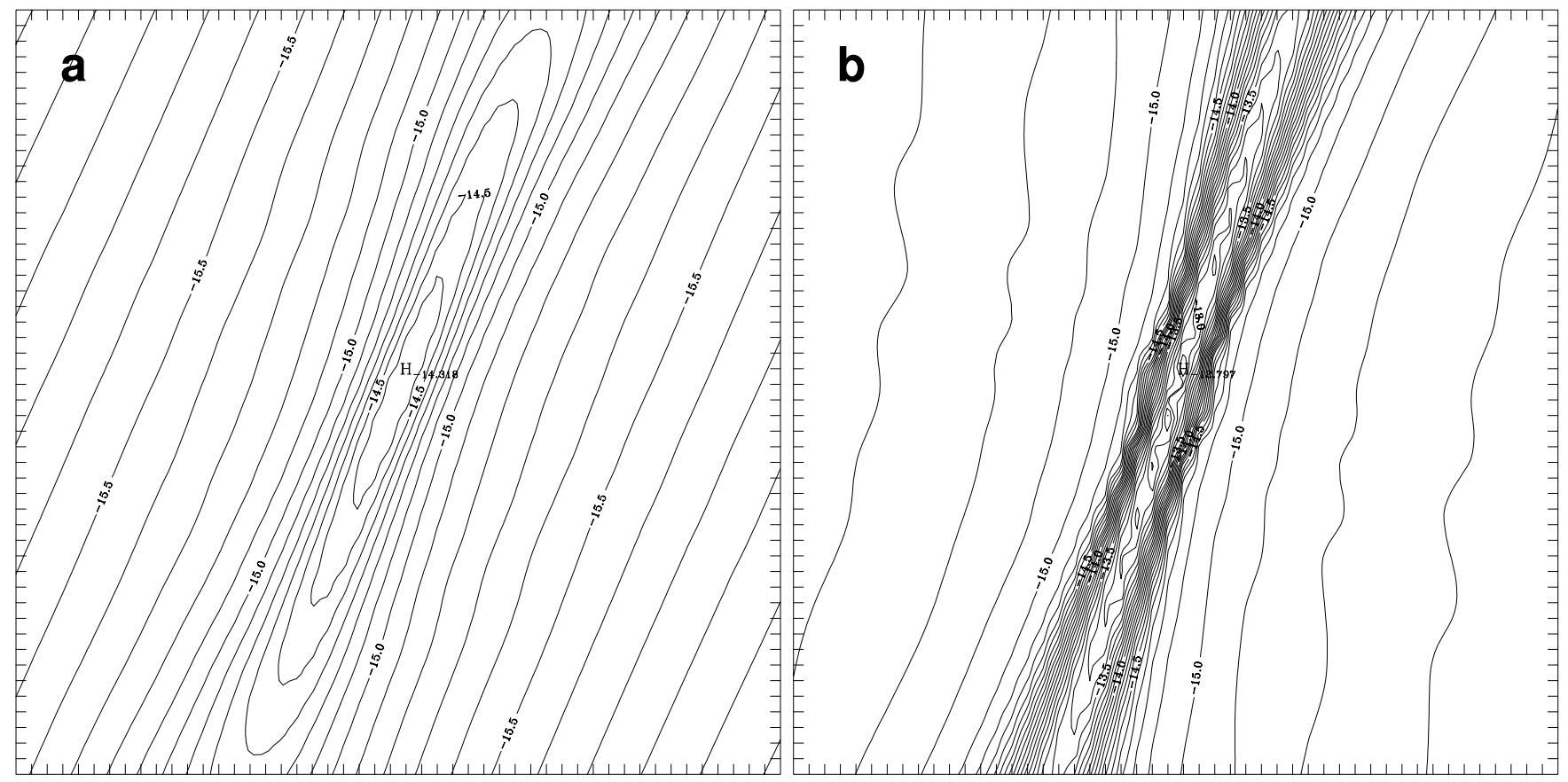

CONTOUR FROM -17 TO -14.3 BY .1

CONTOUR FROM -17 TO -12.8 BY .1

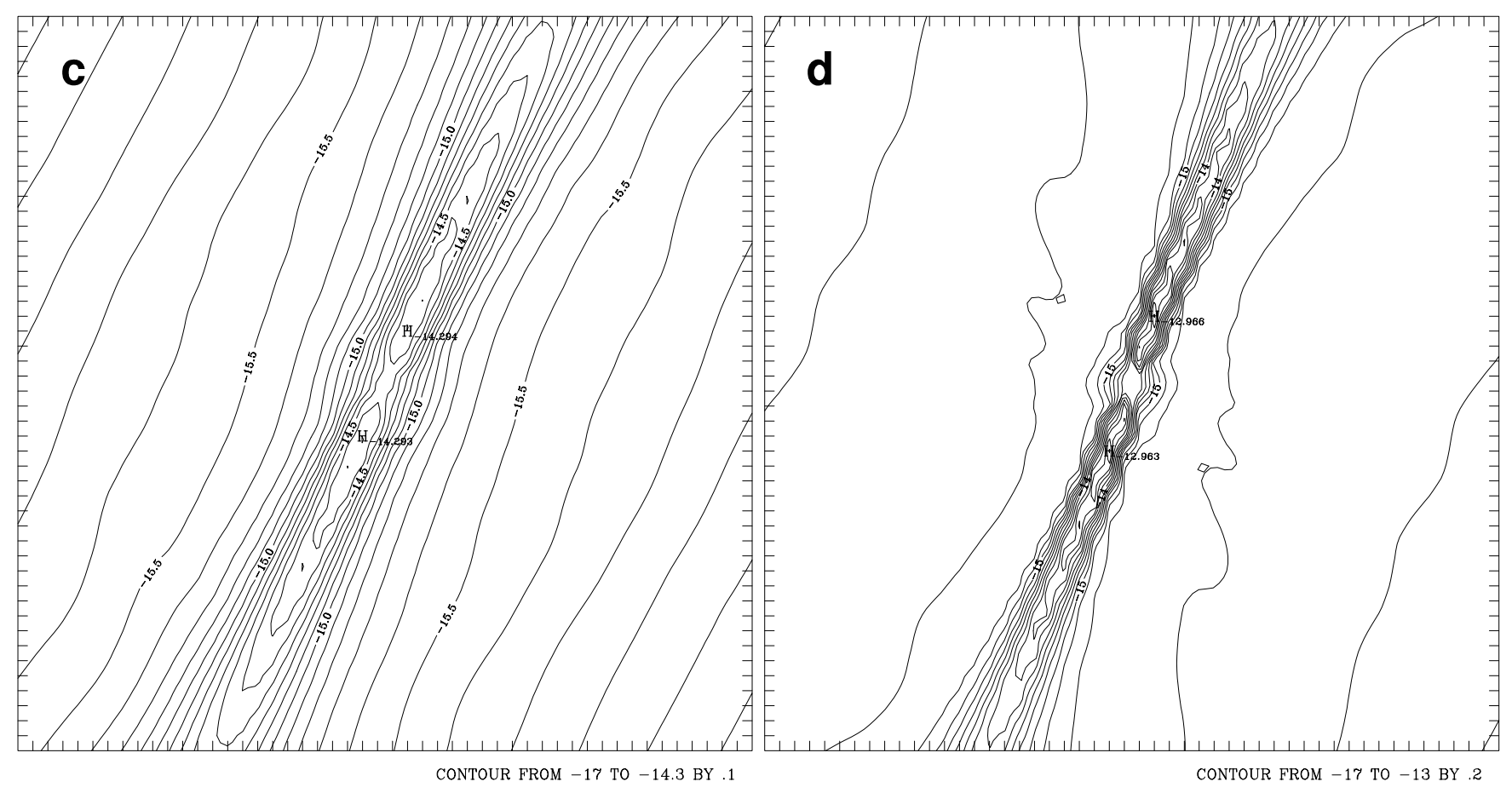

Fig. 7. Density contours in the equatorial plane for the late evolution of model P2, a) and b) with $1+n_{r^{\prime}}=201$ points, and $\mathbf{c})$ and $\mathbf{d}$ ) with $1+n_{r^{\prime}}=51$ points. The times are: a) $1.261 t_{\mathrm{ff}}$, b) $\left.1.265 t_{\mathrm{ff}}, \mathbf{c}\right) 1.263 t_{\mathrm{ff}}$, and d) $1.269 t_{\mathrm{ff}}$. The contour lines correspond to the logarithm of the density. Maximum densities and box radii are: a) $5.40 \times 10^{4} \rho_{0}, 9.0 \times 10^{15} \mathrm{~cm}$. b) $1.66 \times 10^{6} \rho_{0}, 3.0 \times 10^{15} \mathrm{~cm}$. c) $5.12 \times 10^{4} \rho_{0}, 9.0 \times 10^{15} \mathrm{~cm}$. d) $1.18 \times 10^{6} \rho_{0}, 9.0 \times 10^{15} \mathrm{~cm}$. The cloud collapses to produce a singular filament b) in the high-resolution model, and an artificial binary system $\mathbf{d}$ ) in the low-resolution calculation. The magnification in b) implies a radial grid consisting of 6501 points covering the initial cloud radius.

to a singular filament in the same way as occurred for the non-prolate Gaussian cloud collapse model of Sect. 3. Fragmentation is indeed not observed if the mass per unit length of the filaments exceeds its equilibrium value (see Table 1), a result which is consistent with the linear analysis of Inutsuka \& Miyama (1992). The filament region is also depicted in Fig. 8, which shows the equatorial velocity field for model P2 at the same time of Fig. 7b. We note that accretion toward the center of the filament is primarily dominated by low angular momentum mass, while accretion of high angular momentum mass occurs at much larger radii and toward the ends of the filament. 


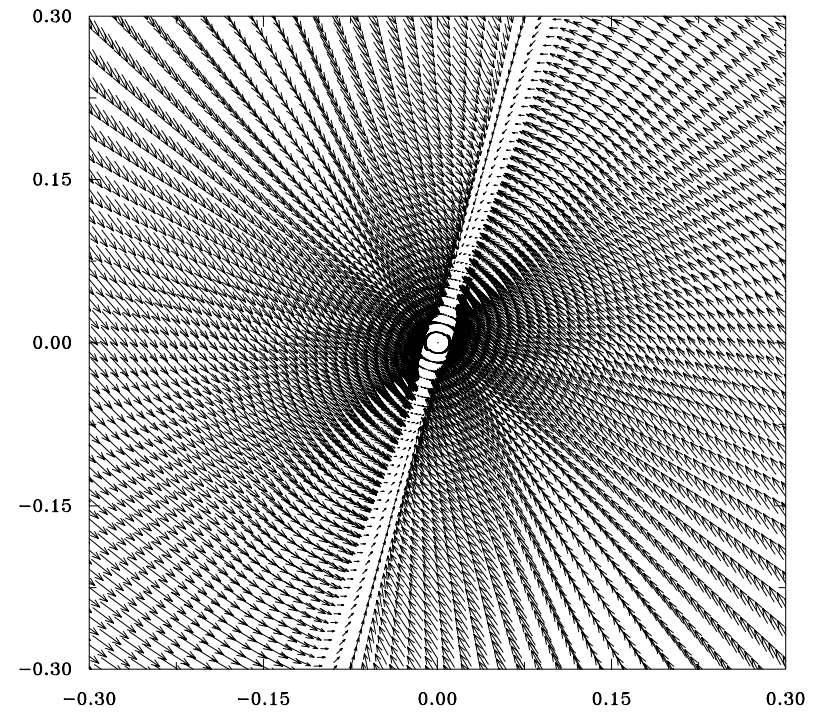

Fig. 8. Velocity vectors in the equatorial plane for model P2 at the same time of Fig. $7 \mathrm{~b}$. A radius of $3.0 \times 10^{15} \mathrm{~cm}$ is shown. The maximum velocity is $0.986 \mathrm{~km} \mathrm{~s}^{-1}$. The velocities are expressed by their values in the ordinary coordinates.

Inside the filament the flow is mainly radially inward and decays significantly toward the center (see Figs. 5c and d).

Figure 9 shows the final outcome obtained for model P3, starting with a much lower rotational energy $(\beta \approx 0.025)$, for both the high (Fig. 9a) and low (Fig. 9b) resolution cases. Again the result diverges, corresponding to a filamentary solution for the $1+n_{r^{\prime}}=201$ calculation and a binary system for the $1+n_{r^{\prime}}=51$ case. Similar results were also obtained for models $\mathrm{P} 4$ and $\mathrm{P} 5$. The innermost filament in Fig. 9a is centrally condensed with two density maxima at each extreme. However, these perturbations never grow because the mass per unit length of the filament exceeds the critical value for equilibrium. The finding that prolate, Gaussian cloud cores are not susceptible to binary fragmentation during their isothermal phase of collapse, along with the need of retardation mechanisms to impede the collapse toward a singularity and allow fragmentation to occur, implies that evaluating the likelihood of fragmentation in prolate cloud cores requires the inclusion of the effects of radiative transfer with appropriate thermodynamics. This is of particular importance because the continued detection of PMS stars strongly suggests that binary fragmentation may be a very frequent process during the early stages of star formation, provided that observations of dense molecular cloud cores continue to show that precollapse clouds often have elongated shapes, moderate central condensations, and values of the thermal, rotational, and gravitational energy appropriate for dynamical collapse.

\section{Conclusions}

New hydrodynamical calculations of the protostellar collapse of centrally condensed, prolate cloud cores, working at very high spatial resolution, have been made to assess
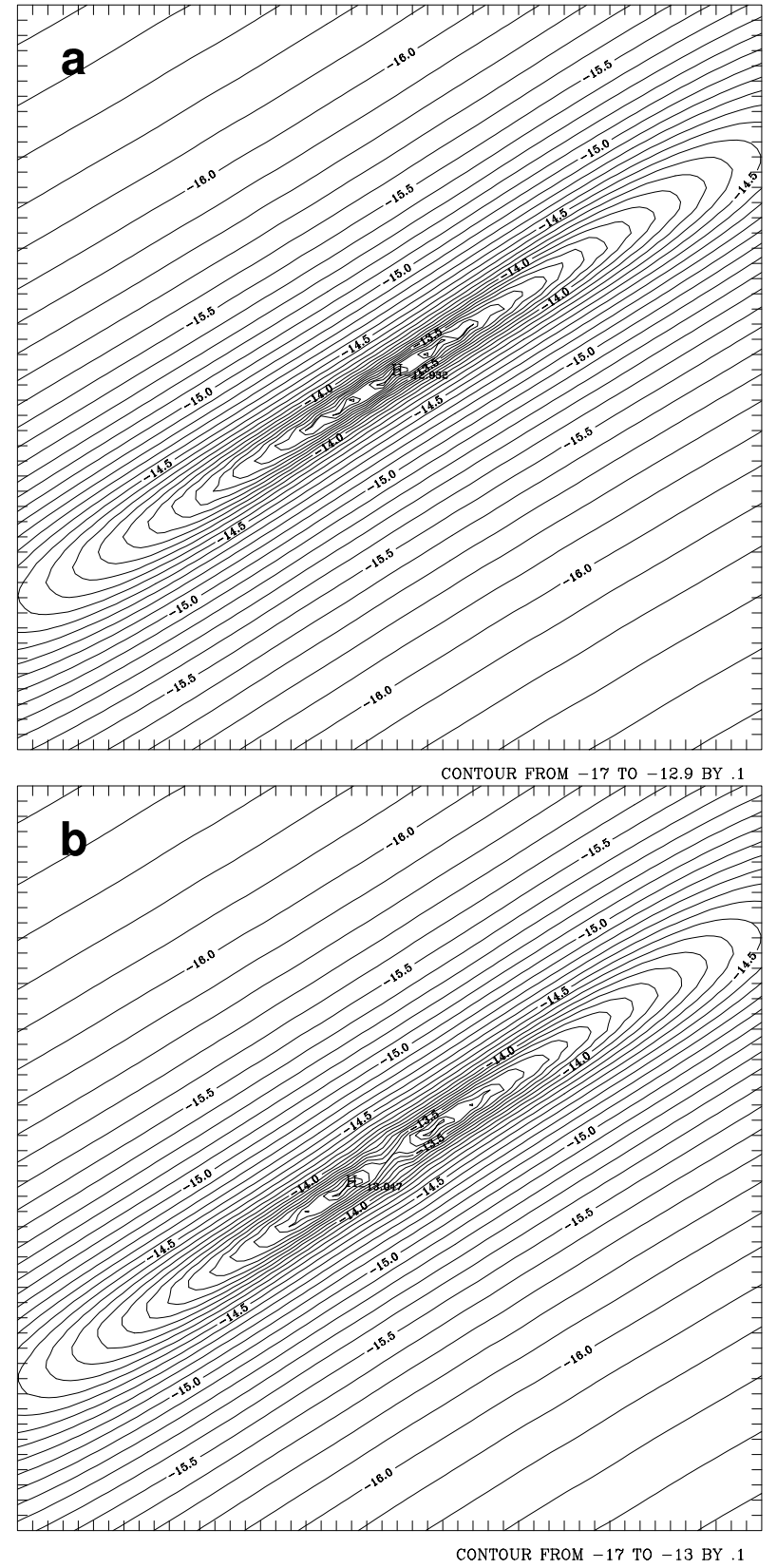

Fig. 9. Density contours in the equatorial plane for the late evolution of model P3, a) with $1+n_{r^{\prime}}=201$ points, and $\mathbf{b}$ ) with $1+n_{r^{\prime}}=51$ points. The times are: a) $1.145 t_{\mathrm{ff}}$ and $\mathbf{b}$ ) $1.146 t_{\mathrm{ff}}$. The contour lines correspond to the logarithm of the density. Maximum densities and box radii are: a) $1.39 \times 10^{6} \rho_{0}$, $8.0 \times 10^{15} \mathrm{~cm}$. b) $1.0 \times 10^{6} \rho_{0}, 8.0 \times 10^{15} \mathrm{~cm}$. The cloud collapses to produce a singular filament a) in the high-resolution model, and an artificial binary system b) in the low-resolution calculation. The magnification in a) implies a radial grid consisting of 5885 points covering the initial cloud radius.

whether elongated configurations collapse isothermally to produce a protostellar binary system, as predicted by previous calculations, or condense into a singular filament without fragmenting, as found by Truelove et al. (1997) and Boss et al. (2000) for the collapse of the spherical, Gaussian cloud model. The initial conditions were chosen to resemble both the elongated molecular cloud cores 
observed by Myers et al. (1991) and Ryden (1996) as well as the central condensation of suspected precollapse cloud clumps (Ward-Thompson et al. 1994; André et al. 1998; Motte et al. 1998). The calculations were made using a spherical-coordinate code which employs zooming coordinates to achieve the required high spatial resolution. These coordinates provide an effective refinement method and guarantees adequate resolution of the local Jeans length during the whole isothermal phase of collapse.

The main conclusions can be summarized as follows:

1. Previous calculations have shown that prolate cloud core models with moderate initial thermal energy $(\alpha \approx 0.22)$ and varied rotational energy $(0.246 \leq \beta \leq$ $0.00025)$ are highly susceptible to fragment into protostellar binary systems during their isothermal collapse phase. However, the outcome of fragmentation for these models has been questioned by the results of Truelove et al. (1997), who introduced the Jeans condition to determine what level of spatial resolution is needed to avoid artificial fragmentation during protostellar collapse calculations. The results of this paper show that working at sufficiently high spatial resolution coupled with the Jeans condition, such prolate cloud core models no longer fragment into binary systems during their isothermal collapse but rather condense to form singular filaments as in the Gaussian cloud model (Truelove et al. 1997; Boss et al. 2000) and the spherical cloud models of Matsumoto \& Hanawa (1999). These results indicate that the outcome of filament formation during the isothermal collapse is essentially insensitive to the initial shape and model conditions.

2. Owing to the initial rotation, the prolate spheroidal cloud first collapses down to the rotational axis producing a flattened, triaxial ellipsoidal figure. During this initial phase, the ellipsoid does not elongate much and so the initial axis ratio is preserved in the equatorial plane of the cloud. A stage of bar formation sets in when mass in the equatorial plane starts collapsing at a significant rate toward the long axis of the cloud. During this phase, the ellipsoid elongates significantly and becomes barlike. Once formed the bar shrinks indefinitely into a singular filament without never fragmenting.

3. The filaments which form possess a mass per unit length $(M / L)$ greater than the equilibrium value $(M / L)_{\mathrm{e}}=2 c_{\mathrm{s}}^{2} / G$, and hence in accordance with the linear analysis of Inutsuka \& Miyama (1992), they will not fragment as long as the collapse remains isothermal. Future calculations may need to employ detailed, radiative transfer solutions to account for the nonisothermal heating. In this physically more realistic case, the gradual heating of the core may retard the collapse leading to fragmentation of the filament into binary or multiple systems.

4. The collapse of the intermediate ellipsoid into a long bar and of the bar into a singular filament occurs selfsimilarly. The self-similar solutions are remarkably similar to those found by Matsumoto et al. (1997) when studying the collapse of a suitably perturbed, rotating, spherical gas cloud into a dynamically contracting disk with subsequent bar formation.

5 . The results may have some implications for binary fragmentation. In all models, the filaments which form are several times longer than the Jeans length. In most cases, the filament forms at maximum densities that are typical of the transition from the isothermal to the nonisothermal phase. As the filament becomes opaque to its own radiation, the increasing pressure forces may retard the collapse and allow the growth of density perturbations along the filament length eventually leading to binary or multiple fragmentation (Bate \& Burkert 1997; Klein et al. 1998; Boss et al. 2000).

Acknowledgements. We thank Andreas Burkert for providing a number of helpful suggestions and comments that have improved the presentation and quality of the manuscript. The calculations of this paper were performed using the IBM Power 3 machine of the Instituto Nacional de Investigaciones Nucleares (ININ) of Mexico and the Origin 2000 machine of the Instituto Mexicano del Petróleo (IMP). This work was supported in part by the venezuelan Consejo Nacional de Investigaciones Científicas y Tecnológicas (CONICIT) and the mexican Consejo Nacional de Ciencias y Tecnología (CONACyT).

\section{References}

André, P., Ward-Thompson, D., \& Motte, F. 1996, A\&A, 314, 625

André, P., Bacmann, A., Motte, F., \& Ward-Thompson, D. 1998, in The Physics and Chemistry of the Interstellar Medium, Proc. 3rd Cologne-Zermatt Symp., ed. V. Ossenkopf, J. Stutzki, \& G. Winnewisser (Zermatt: GCAVerlag), 241

Barranco, J. A., \& Goodman, A. A. 1998, ApJ, 504, 207

Basu, S., \& Mouschovias, T. Ch. 1994, ApJ, 432, 720

Bate, M. R., \& Burkert, A. 1997, MNRAS, 288, 1060

Blottiau, P., Bouquet, S., \& Chièze, J. P. 1988, A\&A, 207, 24

Bodenheimer, P. 1995, ARA\&A, 33, 199

Bodenheimer, P., Burkert, A., Klein, R. I., \& Boss, A. P. 2000, in Protostars and Planets IV, ed. V. G. Mannings, A. P. Boss, \& S. S. Russell (Tucson: Univ. Arizona Press), 675

Bonnell, I. A., Bate, M. R., \& Price, N. M. 1996, MNRAS, 279, 121

Boss, A. P. 1991, Nature, 351, 298

Boss, A. P. 1993, ApJ, 410, 157

Boss, A. P. 1998, ApJ, 501, L77

Boss, A. P., \& Myhill, E. A. 1995, ApJ, 451, 218

Boss, A. P., Fisher, R. T., Klein, R. I., \& McKee, C. F. 2000, ApJ, 528, 325

Bouquet, S., Feix, M. R., Fijalkow, E., \& Munier, A. 1985, ApJ, 293, 494

Burkert, A., \& Bodenheimer, P. 1996, MNRAS, 280, 1190

Curry, C. L. 2000, ApJ, 541, 831

Dutrey, A., Langer, W. D., Bally, J., et al. 1991, A\&A, 270, 468

Fiedler, R. A., \& Mouschovias, T. Ch. 1993, ApJ, 415, 680

Fiege, J., \& Pudritz, R. E. 2000, MNRAS, 311, 105 
Goodman, A. A., Benson, P. J., Fuller, G. A., \& Myers, P. C. 1993, ApJ, 406, 528

Hanawa, T., Nakamura, F., Matsumoto, T., \& Nakano, T. 1994, in Clouds, Cores, and Low Mass Stars, ed. D. P. Clemens, \& R. Barvainis (San Francisco: ASP), ASP Conf. Ser., 65, 151

Heyer, M. H., Vrba, F. J., Snell, R. L., et al. 1987, ApJ, 321, 855

Inutsuka, S.-I., \& Miyama, S. M. 1992, ApJ, 388, 392

Jijina, J., Myers, P. C., \& Adams, F. C. 1999, ApJS, 125, 161

Klapp, J., Sigalotti, L. Di G., \& de Felice, F. 1993, A\&A, 273, 175

Klein, R. I., Fisher, R. T., McKee, C. F., \& Truelove, J. K. 1998, in Numerical Astrophysics, ed. K. Tomisaka, \& S. Miyama (Kluwer: Dordrecht), 131

Matsumoto, T., \& Hanawa, T. 1999, ApJ, 521, 659

Matsumoto, T., Hanawa, T., \& Nakamura, F. 1997, ApJ, 478, 569

Motte, F., André, P., \& Neri, R. 1998, A\&A, 336, 150

Motte, F., \& André, P. 1998, in The Physics and Chemistry of the Interstellar Medium, Proc. 3rd Colgne-Zermatt Symp., 249, ed. V. Ossenkopf, J. Stutzki, \& G. Winnewisser (Zermatt: GCA-Verlag)
Myers, P. C., Fuller, G. A., Goodman, A. A., \& Benson, P. J. 1991, ApJ, 376, 561

Myers, P. C. 1998, in The Physics and Chemistry of the Interstellar Medium, Proc. 3rd Colgne-Zermatt Symp., 227, ed. V. Ossenkopf, J. Stutzki, \& G. Winnewisser (Zermatt: GCA-Verlag)

Munier, A., \& Feix, M. R. 1983, ApJ, 267, 344

Nelson, R. P., \& Papaloizou, J. C. B. 1993, MNRAS, 265, 905

Noh, W. F. 1987, J. Comput. Phys., 72, 78

Onishi, T., Mizuno, A., Kawamura, A., Ogawa, H., \& Fukui, Y. 1996, ApJ, 465, 815

Ryden, B. S. 1996, ApJ, 471, 822

Schneider, S., \& Elmegreen, B. G. 1979, ApJS, 41, 87

Sigalotti, L. Di G. 1998a, ApJ, 498, 236

Sigalotti, L. Di G. 1998b, ApJS, 116, 75

Sigalotti, L. Di G., \& Klapp, J. 1997, ApJ, 474, 710

Sigalotti, L. Di G., \& Klapp, J. 2001, IJMP D, 10(2), 115

Tatematsu, K., Umemoto, T., Kameya, O., et al. 1993, ApJ, 404,643

Truelove, J. K., Klein, R. I., McKee, C. F., et al. 1997, ApJ, 489, L179

Ward-Thompson, D., Scott, P. F., Hills, R. E., \& André, P. 1994, MNRAS, 268, 276 